\title{
An action principle for the Einstein-Weyl equations
}

\author{
Silke Klemm ${ }^{1,2 *}$ and Lucrezia Ravera ${ }^{3,4 \dagger}$ \\ ${ }^{1}$ Dipartimento di Fisica, Università di Milano, Via Celoria 16, 20133 Milano, Italy \\ ${ }^{2}$ INFN, Sezione di Milano, Via Celoria 16, I-20133 Milano, Italy \\ ${ }^{3}$ DISAT, Politecnico di Torino, Corso Duca degli Abruzzi 24, 10129 Torino, Italy \\ ${ }^{4}$ INFN, Sezione di Torino, Via P. Giuria 1, 10125 Torino, Italy
}

June 30, 2020

\begin{abstract}
A longstanding open problem in mathematical physics has been that of finding an action principle for the Einstein-Weyl (EW) equations. In this paper, we present for the first time such an action principle in three dimensions in which the Weyl vector is not exact. More precisely, our model contains, in addition to the Weyl nonmetricity, a traceless part. If the latter is (consistently) set to zero, the equations of motion boil down to the EW equations. In particular, we consider a metric affine $f(R)$ gravity action plus additional terms involving Lagrange multipliers and gravitational Chern-Simons contributions. In our framework, the metric and the connection are considered as independent objects, and no a priori assumptions on the nonmetricity and the torsion of the connection are made. The dynamics of the Weyl vector turns out to be governed by a special case of the generalized monopole equation, which represents a conformal self-duality condition in three dimensions.
\end{abstract}

\footnotetext{
*silke.klemm@mi.infn.it

${ }^{\dagger}$ lucrezia.ravera@polito.it
} 


\section{Introduction}

A remarkable generalization of Riemannian geometry was first proposed in 1918 by Weyl (see e.g. [1-4]), who introduced an additional symmetry in an attempt of geometrically unifying electromagnetism with gravity [5,6]. In this theory, both the direction and the length of vectors are allowed to vary under parallel transport ${ }^{1}$. The trace part of the connection introduced by Weyl is known as the Weyl vector. When it is exact, it can be gauged away by a local scale transformation. In this case, Weyl geometry is said to be integrable (parallel transported vectors along closed paths return with unaltered lengths), and there exists a subclass of global gauges in which the geometry is Riemannian.

Mathematically, a Weyl structure on an $n$-dimensional manifold $\mathcal{M}$ consists of a conformal structure $[g]=\left\{f g, f: \mathcal{M} \rightarrow \mathbb{R}^{+}\right\}$, together with a torsion-free connection $\nabla$ which is compatible with $[g]$ in the sense that

$$
\nabla_{\mu} g_{\nu \rho}=2 h_{\mu} g_{\nu \rho}
$$

for some one-form $h$ on $\mathcal{M}$. This compatibility condition is invariant under the transformation

$$
g \mapsto e^{2 \Omega} g, \quad h \mapsto h+d \Omega,
$$

where $\Omega$ is a function on $\mathcal{M}$.

A Weyl structure is said to be Einstein-Weyl (EW) [8] if the symmetrized Ricci tensor $R_{\mu \nu}$ of $\nabla$ is proportional to some metric $g \in[g]$. This conformally invariant condition is equivalent to

$$
R_{(\mu \nu)}-\frac{R}{n} g_{\mu \nu}=0
$$

with $R$ the Ricci scalar of $\nabla$.

A longstanding and still unresolved open problem has been that of finding an action principle for (3). Here we shall present for the first time an action principle for the EW equations in $2+1$ dimensions ${ }^{2}$ in which the Weyl vector is not exact. Notice that three-dimensional EW geometry [9] is particularly interesting, since it is related to dispersionless integrable systems [10-12]. Moreover, Jones and Tod [13] showed that selfdual conformal four-manifolds with a conformal vector field are in correspondence with abelian monopoles on Einstein-Weyl three-manifolds. In ref. [14], Gauduchon and Tod studied the structure of four-dimensional hyper-Hermitian Riemannian spaces admitting a tri-holomorphic Killing vector, i.e., a Killing vector that is compatible with the three complex structures on the hyper-Hermitian space. It turned out that the latter is fibered over a specific type of three-dimensional EW spaces, called hyper-CR or Gauduchon-Tod.

Note also that $3 \mathrm{~d}$ EW manifolds and their generalizations play an important role also in high energy physics, for instance in the context of supersymmetric solutions to fake supergavity [15-17], in the classification of four-dimensional Euclidean gravitational instantons [18,19], or supersymmetric near-horizon geometries [20,21]. Moreover, Weyl connections were considered recently in holography [22]. For a general review of Einstein manifolds with nonmetric and torsionful connections cf. [23].

\footnotetext{
${ }^{1}$ Note, however, that Weyl's attempt to identify the trace part of the connection, associated with stretching and contraction, with the vector potential of electromagnetism failed, due to observational inconsistencies [7].

${ }^{2}$ Our results hold also in three Euclidean dimensions.
} 
Our construction of an action that leads to (3) involves a metric affine $f(R)$ gravity action [24-29] plus additional terms containing Lagrange multipliers and gravitational Chern-Simons contributions. We work in a first order formalism, where the metric and the connection are treated as independent variables, and make no a priori assumptions on the metricity and the torsion of the connection.

The remainder of this paper is organized as follows: In section 2 we briefly review metric affine $f(R)$ gravity in $n$ dimensions, following [25,26]. Subsequently, in section 3 we construct an action that yields the EW equations with nonexact Weyl vector in three dimensions. We conclude our work with some final remarks.

\section{Metric affine $f(R)$ gravity in $n$ dimensions}

Consider the gravitational action

$$
S_{\mathrm{G}}=\frac{1}{2 \kappa^{2}} \int d^{n} x \sqrt{-g} f(R)
$$

where $\kappa$ denotes the gravitational coupling constant and $f(R)$ is an arbitrary function of the scalar curvature $R=g^{\mu \nu} R_{\mu \nu}(\Gamma)$, with $\Gamma$ a general affine connection ${ }^{3}$. We work in a first order (Palatini) formalism, where the metric $g_{\mu \nu}$ and the connection $\Gamma_{\mu \nu}^{\lambda}$ are treated as independent variables. Variation of (4) w.r.t. $g^{\mu \nu}$ gives

$$
f^{\prime}(R) R_{(\mu \nu)}-\frac{1}{2} f(R) g_{\mu \nu}=0,
$$

while the variation w.r.t. $\Gamma^{\lambda}{ }_{\mu \nu}$ leads to

$$
\begin{aligned}
& \frac{1}{\sqrt{-g}}\left[-\nabla_{\lambda}\left(\sqrt{-g} f^{\prime}(R) g^{\mu \nu}\right)+\nabla_{\sigma}\left(\sqrt{-g} f^{\prime}(R) g^{\mu \sigma}\right) \delta_{\lambda}^{\nu}\right] \\
& +2 f^{\prime}(R)\left(g^{\mu \nu} \Gamma_{[\lambda \sigma]}^{\sigma}-g^{\mu \rho} \Gamma_{[\rho \sigma]}^{\sigma} \delta_{\lambda}{ }_{\lambda}+g^{\mu \sigma} \Gamma_{[\sigma \lambda]}^{\nu}\right)=0 .
\end{aligned}
$$

The trace of (5) yields

$$
\frac{f}{2 f^{\prime}}=\frac{R}{n}
$$

which is identically satisfied if we choose

$$
f=C R^{n / 2},
$$

where $C$ is an arbitrary integration constant. Let us observe that (7) could also be viewed as an algebraic equation on $R$ admitting generically solutions with constant scalar curvature (cf. e.g. [26]). Here, we consider the specific choice (8). Then (with $C=1$ ), the action (4) becomes

$$
S_{\mathrm{G}}=\frac{1}{2 \kappa^{2}} \int d^{n} x \sqrt{-g} R^{n / 2}
$$

which is invariant under the conformal transformation (we use the same definition as [26])

$$
g_{\mu \nu} \mapsto g_{\mu \nu}^{\prime}=e^{2 \Omega} g_{\mu \nu}, \quad \Gamma_{\mu \nu}^{\lambda} \mapsto \Gamma_{\mu \nu}^{\prime \lambda}=\Gamma_{\mu \nu}^{\lambda},
$$

\footnotetext{
${ }^{3}$ We adopt the same conventions of [25,26]. In particular, our metric convention is $\eta=\operatorname{diag}(-,+,+)$.
} 
where $\Omega$ is a scalar function. Indeed, under (10) we have

$$
d^{n} x \sqrt{-g} \mapsto d^{n} x e^{n \Omega} \sqrt{-g}, \quad R_{\mu \nu \rho}^{\lambda} \mapsto R_{\mu \nu \rho}^{\lambda}, \quad R_{\mu \rho} \mapsto R_{\mu \rho}, \quad R \mapsto e^{-2 \Omega} R,
$$

and one can clearly see that (9) is invariant.

Now, plugging (7) into (5), the latter boils down to

$$
R_{(\mu \nu)}-\frac{R}{n} g_{\mu \nu}=0
$$

In the case in which one has Weyl nonmetricity (1) and vanishing torsion, (12) precisely corresponds to the Einstein-Weyl equations. Observe that (12) is traceless.

Let us take a closer look at (6). Using the definition of the Cartan torsion tensor,

$$
T_{\mu \nu}^{\rho}:=\Gamma_{[\mu \nu]}^{\rho},
$$

(6) can be rewritten as

$$
\begin{aligned}
& \frac{1}{\sqrt{-g}}\left[-\nabla_{\lambda}\left(\sqrt{-g} f^{\prime}(R) g^{\mu \nu}\right)+\nabla_{\sigma}\left(\sqrt{-g} f^{\prime}(R) g^{\mu \sigma}\right) \delta_{\lambda}^{\nu}\right] \\
& +2 f^{\prime}(R)\left(g^{\mu \nu} T_{\lambda}-T^{\mu} \delta^{\nu}{ }_{\lambda}+g^{\mu \sigma} T_{\sigma \lambda}{ }^{\nu}\right)=0
\end{aligned}
$$

where $T_{\lambda}:=T_{\lambda \sigma}{ }^{\sigma}$ is the trace part of the torsion. Taking the $\lambda, \mu$ trace of (14) leads to the identity

$$
P_{\mu}^{\mu \nu}=0,
$$

where we introduced the so-called Palatini tensor,

$$
P_{\lambda}^{\mu \nu}=-\frac{\nabla_{\lambda}\left(\sqrt{-g} g^{\mu \nu}\right)}{\sqrt{-g}}+\frac{\nabla_{\sigma}\left(\sqrt{-g} g^{\mu \sigma}\right) \delta^{\nu}}{\sqrt{-g}}+2\left(g^{\mu \nu} T_{\lambda}-T^{\mu} \delta_{\lambda}^{\nu}+g^{\mu \sigma} T_{\sigma \lambda}{ }^{\nu}\right),
$$

which is indeed traceless. Contracting $\lambda$ and $\nu$ in (14), one obtains ${ }^{4}$

$$
(n-1) \nabla_{\sigma}\left(\sqrt{-g} f^{\prime}(R) g^{\sigma \mu}\right)=2(n-2) \sqrt{-g} f^{\prime}(R) T^{\mu} .
$$

Plugging (17) into (14) and taking the symmetric and antisymmetric part in $\mu, \nu$, we get respectively

$$
\left.-\frac{1}{\sqrt{-g}} \nabla_{\lambda}\left(\sqrt{-g} f^{\prime}(R) g^{\mu \nu}\right)+2 f^{\prime}(R)\left(g^{\mu \nu} T_{\lambda}-\frac{1}{n-1} g^{\rho(\mu} \delta_{\lambda}^{\nu)} T_{\rho}+g^{\sigma(\mu} T_{\sigma \lambda} \nu\right)\right)=0,
$$

and

$$
-\frac{1}{n-1} g^{\rho[\mu} \delta_{\lambda}^{\nu]} T_{\rho}+g^{\sigma[\mu} T_{\sigma \lambda}^{\nu]}=0 .
$$

After some algebraic manipulation, (19) gives

$$
T_{\lambda \mu}{ }^{\nu}=\frac{2}{n-1} T_{[\lambda} \delta_{\mu]}{ }^{\nu},
$$

\footnotetext{
${ }^{4}$ Here we also fix a miscalculation appearing in [27].
} 
i.e., the torsion is completely determined by its trace part $T_{\lambda}$. On the other hand, if we take the $\mu, \nu$ trace in (18) and use the general formula $[25,26]$

$$
\nabla_{\lambda} \sqrt{-g}=-\frac{1}{2} \sqrt{-g} g_{\mu \nu} \nabla_{\lambda} g^{\mu \nu}=-\frac{1}{2} \sqrt{-g} g_{\mu \nu} Q_{\lambda}{ }^{\mu \nu}=-\frac{1}{2} \sqrt{-g} Q_{\lambda},
$$

where $Q_{\lambda} \equiv Q_{\lambda \mu}{ }^{\mu}$ is a trace of the nonmetricity tensor ${ }^{5}$, we find

$$
\frac{n-2}{2 n} Q_{\lambda}+\frac{2(n-2)}{n-1} T_{\lambda}=\frac{\partial_{\lambda} f^{\prime}}{f^{\prime}}
$$

or equivalently

$$
\frac{n-2}{2} w_{\lambda}=\partial_{\lambda} \ln f^{\prime}
$$

where we introduced the so-called affine vector $w_{\lambda}$ [25], defined in $n$ dimensions by

$$
w_{\lambda}:=\frac{1}{n} Q_{\lambda}+\frac{4}{n-1} T_{\lambda} .
$$

Observe that, using (8), eq. (23) can be rewritten as

$$
\partial_{\lambda} \ln R=w_{\lambda},
$$

i.e., the affine vector is exact. Now, plugging the expression for $\partial_{\lambda} \ln f^{\prime}$ from (22) and the torsion (20) into (18), we obtain

$$
Q_{\lambda \mu \nu}=\frac{1}{n} Q_{\lambda} g_{\mu \nu}
$$

The nonmetricity is thus fully determined by the vector $Q_{\lambda}$. Notice that in the irreducible decomposition of the nonmetricity tensor under the Lorentz group there is a second nonmetricity vector $\tilde{Q}_{\nu}:=Q^{\mu}{ }_{\mu \nu}$. Using (26), one gets $\tilde{Q}_{\nu}=\frac{1}{n} Q_{\nu}$, and therefore (26) can alternatively be written as $Q_{\lambda \mu \nu}=\tilde{Q}_{\lambda} g_{\mu \nu}$.

Finally, exploiting the generic decomposition of an affine connection,

$$
\Gamma_{\mu \nu}^{\lambda}=\tilde{\Gamma}_{\mu \nu}^{\lambda}+N_{\mu \nu}^{\lambda},
$$

where the distortion tensor $N^{\lambda}{ }_{\mu \nu}$ and the Levi-Civita connection $\tilde{\Gamma}_{\mu \nu}^{\lambda}$ are respectively given by

$$
\begin{gathered}
N_{\mu \nu}^{\lambda}=\underbrace{\frac{1}{2} g^{\rho \lambda}\left(Q_{\mu \nu \rho}+Q_{\nu \rho \mu}-Q_{\rho \mu \nu}\right)}_{\text {deflection }}-\underbrace{g^{\rho \lambda}\left(T_{\rho \mu \nu}+T_{\rho \nu \mu}-T_{\mu \nu \rho}\right)}_{\text {contorsion }} \\
\tilde{\Gamma}_{\mu \nu}^{\lambda}=\frac{1}{2} g^{\rho \lambda}\left(\partial_{\mu} g_{\nu \rho}+\partial_{\nu} g_{\rho \mu}-\partial_{\rho} g_{\mu \nu}\right)
\end{gathered}
$$

one can show that, in the present case, the complete expression for the affine connection reads

$$
\Gamma_{\mu \nu}^{\lambda}=\tilde{\Gamma}_{\mu \nu}^{\lambda}+\frac{1}{2 n} Q_{\nu} \delta_{\mu}^{\lambda}+\frac{1}{2}\left(w_{\mu} \delta_{\nu}^{\lambda}-g_{\mu \nu} w^{\lambda}\right) .
$$

\footnotetext{
${ }^{5}$ The latter is defined by $Q_{\lambda \mu \nu}:=-\nabla_{\lambda} g_{\mu \nu}=-\partial_{\lambda} g_{\mu \nu}+\Gamma_{\mu \lambda}^{\rho} g_{\rho \nu}+\Gamma^{\rho}{ }_{\nu \lambda} g_{\mu \rho}$.
} 
Notice that, in $f(R)$ theories, the connection is only determined up to a vectorial degree of freedom $[25,26]$. Indeed, using the definition of the Ricci tensor,

$$
R_{\mu \nu}:=R_{\mu \lambda \nu}^{\lambda}=2 \partial_{[\lambda} \Gamma_{|\mu| \nu]}^{\lambda}+2 \Gamma_{\rho[\lambda}^{\lambda} \Gamma_{|\mu| \nu]}^{\rho},
$$

one can show that under projective transformations ${ }^{6}$

$$
\Gamma_{\mu \nu}^{\lambda} \mapsto \Gamma_{\mu \nu}^{\prime \lambda}=\Gamma_{\mu \nu}^{\lambda}+\delta_{\mu}^{\lambda} \xi_{\nu}
$$

where $\xi_{\nu}$ is an arbitrary vector field, the symmetric part of the Ricci tensor and thus the Ricci scalar and the action (4) remain invariant.

Observe that in the case in which (7) is considered as a purely algebraic equation for the scalar curvature $R$ yielding constant curvature metrics, one has, from (25), that the affine vector $w_{\mu}$ vanishes, which implies

$$
Q_{\mu}=-\frac{4 n}{n-1} T_{\mu}
$$

and

$$
\Gamma_{\mu \nu}^{\lambda}=\tilde{\Gamma}_{\mu \nu}^{\lambda}+\frac{1}{2 n} Q_{\nu} \delta_{\mu}^{\lambda} .
$$

One can therefore always arrive at the Levi-Civita connection by choosing $\xi_{\nu}$ appropriately in (32).

On the other hand, in the case of $f(R)$ theories with $f(R)=R^{n / 2}$, taking into account that under the projective transformation (32) one has ${ }^{7}$

$$
\begin{array}{ll}
T_{\mu \nu}^{\lambda} \mapsto T_{\mu \nu}^{\lambda}+\delta_{[\mu}^{\lambda} \xi_{\nu]}, & T_{\mu} \mapsto T_{\mu}-\frac{n-1}{2} \xi_{\mu}, \\
N^{\lambda}{ }_{\mu \nu} \mapsto N^{\lambda}{ }_{\mu \nu}+\delta^{\lambda} \xi_{\nu}, & Q_{\lambda \mu \nu} \mapsto Q_{\lambda \mu \nu}+2 \xi_{\lambda} g_{\mu \nu}, \quad Q_{\mu} \mapsto Q_{\mu}+2 n \xi_{\mu},
\end{array}
$$

one easily shews that the affine vector $w_{\mu}$ is invariant under (32). This is consistent with the fact that $w_{\mu}$ is related by (25) to the scalar curvature $R$, which was shown above to be projectively invariant. One can use the projective gauge freedom to further restrict the torsion and nonmetricity, and thus the affine connection. For instance, we can choose $\xi_{\mu}$ in such a way to eliminate either the torsion or the nonmetricity [25,26],

$$
\begin{aligned}
& \xi_{\mu}=\frac{2}{n-1} T_{\mu} \Rightarrow\left\{\begin{array}{l}
T_{\mu}^{\prime}=0 \Rightarrow T_{\mu \nu}^{\prime \lambda}=0, \\
Q_{\mu}^{\prime}=Q_{\mu}+\frac{4 n}{n-1} T_{\mu} \Rightarrow Q_{\lambda \mu \nu}^{\prime}=Q_{\lambda \mu \nu}+\frac{4}{n-1} T_{\lambda} g_{\mu \nu} ;
\end{array}\right. \\
& \xi_{\mu}=-\frac{1}{2 n} Q_{\mu} \Rightarrow\left\{\begin{array}{l}
T_{\mu}^{\prime}=T_{\mu}+\frac{n-1}{4 n} Q_{\mu} \Rightarrow T_{\mu \nu}^{\prime \lambda}=T_{\mu \nu}{ }^{\lambda}+\frac{1}{2 n} Q_{[\mu} \delta_{\nu]}{ }^{\lambda}, \\
Q_{\mu}^{\prime}=0 \Rightarrow Q_{\lambda \mu \nu}^{\prime}=0 .
\end{array}\right.
\end{aligned}
$$

Intriguingly, one may also choose $\xi_{\mu}$ in such a way that the torsion and nonmetricity vectors result to be interchanged $[25]^{8}$,

$$
\xi_{\mu}=\frac{2}{n-1} T_{\mu}-\frac{1}{2 n} Q_{\mu} \Rightarrow\left\{\begin{array}{l}
T_{\mu}^{\prime}=\frac{n-1}{4 n} Q_{\mu} \Rightarrow T_{\mu \nu}^{\prime \lambda}=\frac{1}{2 n} Q_{[\mu} \delta_{\nu]}{ }^{\lambda}, \\
Q_{\mu}^{\prime}=\frac{4 n}{n-1} T_{\mu} \Rightarrow Q_{\lambda \mu \nu}^{\prime}=\frac{4}{n-1} T_{\lambda} g_{\mu \nu} .
\end{array}\right.
$$

\footnotetext{
${ }^{6}$ The latter are defined as those transformations of the affine connection that leave the autoparallels of vectors invariant up to reparametrizations of the affine parameter [25].

${ }^{7}$ Here we use $N_{(\lambda \mu) \nu}=\frac{1}{2} Q_{\nu \lambda \mu}$.

${ }^{8}$ Notice that, in this case, the scalar product $T_{\mu} Q^{\mu}$ is left invariant under the transformation.
} 
In this sense, systems with spacetime dislocations (induced by torsion) are physically equivalent to systems with Weyl nonmetricity [25].

As we already mentioned, the action (9) is also invariant under the conformal transformations (10), under which the torsion and nonmetricity transform respectively as

$$
T_{\mu \nu}^{\lambda} \mapsto T_{\mu \nu}^{\prime \lambda}=T_{\mu \nu}^{\lambda}, \quad Q_{\lambda \mu \nu} \mapsto Q_{\lambda \mu \nu}^{\prime}=e^{2 \Omega}\left[Q_{\lambda \mu \nu}-2 g_{\mu \nu} \partial_{\lambda} \Omega\right],
$$

which implies

$$
T_{\mu} \mapsto T_{\mu}^{\prime}=T_{\mu}, \quad Q_{\mu} \mapsto Q_{\mu}^{\prime}=Q_{\mu}-2 n \partial_{\mu} \Omega, \quad \tilde{Q}_{\mu} \mapsto \tilde{Q}_{\mu}^{\prime}=\tilde{Q}_{\mu}-2 \partial_{\mu} \Omega .
$$

For further details on transformations in metric affine manifolds we refer to [26].

To conclude this preliminary review, let us now briefly discuss, following [26], what happens when one adds a matter action $S_{\mathrm{M}}\left[g_{\mu \nu}, \Gamma_{\mu \nu}^{\lambda}\right]$ to (4). The full action is then

$$
S=S_{\mathrm{G}}+S_{\mathrm{M}} \text {. }
$$

Variation of (40) w.r.t. $g^{\mu \nu}$ gives

$$
f^{\prime}(R) R_{(\mu \nu)}-\frac{1}{2} f(R) g_{\mu \nu}=\kappa^{2} T_{\mu \nu}
$$

where

$$
T_{\mu \nu}:=-\frac{2}{\sqrt{-g}} \frac{\delta S_{\mathrm{M}}}{\delta g^{\mu \nu}}
$$

is the energy-momentum tensor ${ }^{9}$, while the variation w.r.t. $\Gamma^{\lambda}{ }_{\mu \nu}$ leads to

$$
\begin{aligned}
& \frac{1}{\sqrt{-g}}\left[-\nabla_{\lambda}\left(\sqrt{-g} f^{\prime}(R) g^{\mu \nu}\right)+\nabla_{\sigma}\left(\sqrt{-g} f^{\prime}(R) g^{\mu \sigma}\right) \delta_{\lambda}^{\nu}\right] \\
& +2 f^{\prime}(R)\left(g^{\mu \nu} \Gamma_{[\lambda \sigma]}^{\sigma}-g^{\mu \rho} \Gamma_{[\rho \sigma]}^{\sigma} \delta_{\lambda}^{\nu}+g^{\mu \sigma} \Gamma_{[\sigma \lambda]}^{\nu}\right)=\kappa^{2} \Delta_{\lambda}{ }^{\mu \nu},
\end{aligned}
$$

with the hypermomentum tensor

$$
\Delta_{\lambda}{ }^{\mu \nu} \equiv-\frac{2}{\sqrt{-g}} \frac{\delta S_{\mathrm{M}}}{\delta \Gamma^{\lambda}{ }_{\mu \nu}}
$$

that contains information on the spin, shear and dilation of matter. Using the Cartan torsion tensor, (43) can be cast into

$$
\begin{aligned}
& \frac{1}{\sqrt{-g}}\left[-\nabla_{\lambda}\left(\sqrt{-g} f^{\prime}(R) g^{\mu \nu}\right)+\nabla_{\sigma}\left(\sqrt{-g} f^{\prime}(R) g^{\mu \sigma}\right) \delta^{\nu}{ }_{\lambda}\right] \\
& +2 f^{\prime}(R)\left(g^{\mu \nu} T_{\lambda}-T^{\mu} \delta_{\lambda}^{\nu}+g^{\mu \sigma} T_{\sigma \lambda}{ }^{\nu}\right)=\kappa^{2} \Delta_{\lambda}{ }^{\mu \nu} .
\end{aligned}
$$

The $\lambda, \mu$ trace of (45) gives

$$
\Delta_{\mu}^{\mu \nu}=0,
$$

which cannot hold for any form of matter. (46) arises due to the projective invariance of the Ricci scalar. Metric affine $f(R)$ theories of gravity can thus be consistently coupled only to projectively invariant matter. Note in this context that the terms added to (9) in the next section break projective invariance, so that the resulting theory can be coupled to any type of matter.

\footnotetext{
${ }^{9}$ If $f(R)$ is given by (8), the trace of (41) yields $g^{\mu \nu} T_{\mu \nu}=0$, so this specific model can be coupled only to conformally invariant matter.
} 


\section{An action principle for the Einstein-Weyl equations in three dimensions}

In this section, we present an action principle for the Einstein-Weyl equations in $2+1$ dimensions. To this end, we consider the $f(R)$ contribution (9) plus additional terms involving Lagrange multipliers and gravitational Chern-Simons contributions.

Let us first recall the decomposition of the nonmetricity $Q_{\lambda \mu \nu}$ and torsion $T_{\lambda \mu}{ }^{\nu}$ in a trace and traceless part. In three dimensions, one has [26]

$$
\begin{aligned}
Q_{\lambda \mu \nu} & =\frac{2}{5} Q_{\lambda} g_{\mu \nu}-\frac{1}{5} \tilde{Q}_{\lambda} g_{\mu \nu}+\frac{3}{5} g_{\lambda(\nu} \tilde{Q}_{\mu)}-\frac{1}{5} g_{\lambda(\nu} Q_{\mu)}+\Omega_{\lambda \mu \nu}, \\
T_{\lambda \mu}{ }^{\nu} & =\delta_{[\mu}{ }^{\nu} T_{\lambda]}+S_{\lambda \mu}{ }^{\nu},
\end{aligned}
$$

where the traces $Q_{\lambda}$ (the Weyl vector), $\tilde{Q}_{\lambda}$ and $T_{\lambda}$ were defined in the previous section, while $\Omega_{\lambda \mu \nu}$ and $S_{\lambda \mu}{ }^{\nu}$ denote the traceless parts of the nonmetricity and torsion respectively. In the following, we shall need the variation of the latter w.r.t. the metric and the connection, which is given by [26]

$$
\delta_{g} Q_{\rho \alpha \beta}=\partial_{\rho}\left(g_{\mu \alpha} g_{\nu \beta} \delta g^{\mu \nu}\right)-2 g_{\lambda \mu} g_{\nu(\alpha} \Gamma_{\beta) \rho}^{\lambda} \delta g^{\mu \nu}, \quad \delta_{g} T_{\mu \nu}^{\alpha}=0,
$$

and

$$
\delta_{\Gamma} Q_{\rho \alpha \beta}=2 \delta_{\rho}^{\nu} \delta_{(\alpha}^{\mu} g_{\beta) \lambda} \delta \Gamma^{\lambda}{ }_{\mu \nu}, \quad \delta_{\Gamma} T_{\alpha \beta}^{\lambda}=\delta_{\alpha}^{[\mu} \delta_{\beta}^{\nu]} \delta \Gamma^{\lambda}{ }_{\mu \nu}
$$

These imply

$$
\begin{aligned}
& \delta_{g} Q_{\rho}=\partial_{\rho}\left(g_{\mu \nu} \delta g^{\mu \nu}\right), \quad \delta_{g} T_{\mu}=0, \\
& \delta_{g} \tilde{Q}_{\beta}=\delta g^{\mu \nu}\left[g_{\nu \beta} g^{\rho \alpha} \partial_{\rho} g_{\mu \alpha}+\Gamma_{\mu \nu}^{\lambda} g_{\lambda \beta}-g^{\rho \sigma} \Gamma_{\rho \sigma}^{\alpha} g_{\mu \alpha} g_{\nu \beta}\right]+g_{\nu \beta} \partial_{\mu} \delta g^{\mu \nu}
\end{aligned}
$$

and

$$
\delta_{\Gamma} Q_{\rho}=2 \delta_{\rho}^{\nu} \delta_{\lambda}^{\mu} \delta \Gamma^{\lambda}{ }_{\mu \nu}, \quad \delta_{\Gamma} \tilde{Q}_{\beta}=\left(g^{\mu \nu} g_{\beta \lambda}+\delta_{\beta}^{\mu} \delta_{\lambda}^{\nu}\right) \delta \Gamma_{\mu \nu}^{\lambda}, \quad \delta_{\Gamma} T_{\alpha}=\delta_{\alpha}^{[\mu} \delta_{\lambda}^{\nu]} \delta \Gamma_{\mu \nu}^{\lambda} .
$$

We propose the action

$$
\begin{aligned}
S & =\frac{1}{2 \kappa^{2}} \int d^{3} x\left[\sqrt{-g} f(R)+\frac{1}{2 \mu} \epsilon^{\mu \nu \rho} Q_{\rho} \hat{R}_{\nu \mu}\right]+\int d^{3} x \epsilon^{\mu \nu \rho}\left[\chi_{\rho \mu}\left(-\frac{1}{3} Q_{\nu}+\tilde{Q}_{\nu}\right)+\zeta_{\nu \sigma} T_{\rho \mu}{ }^{\sigma}\right] \\
& +\frac{3}{\kappa^{2} \mu} \int d^{3} x \epsilon^{\mu \nu \rho}\left(\Gamma_{\tau \rho}^{\sigma} \partial_{\mu} \Gamma^{\tau}{ }_{\sigma \nu}+\frac{2}{3} \Gamma_{\sigma \mu}^{\tau} \Gamma_{\alpha \nu}^{\sigma} \Gamma_{\tau \rho}^{\alpha}\right)
\end{aligned}
$$

where $f(R)=R^{3 / 2}, \hat{R}_{\mu \nu}:=R_{\lambda \mu \nu}^{\lambda}=\partial_{[\mu} Q_{\nu]}$ denotes the homothetic curvature tensor, and $\mu$ is a Chern-Simons coupling constant. Note that (54) contains a Chern-Simons term both for the Weyl vector and the connection $\Gamma$. In (54) we also introduced the Levi-Civita symbol $\epsilon^{\mu \nu \rho}=\sqrt{-g} \varepsilon^{\mu \nu \rho}$, where $\varepsilon^{\mu \nu \rho}$ is the Levi-Civita tensor. $\chi_{\mu \nu}=-\chi_{\nu \mu}$ and $\zeta_{\mu \nu}$ are Lagrange multipliers ${ }^{10}$. The idea that gravitational Chern-Simons terms may be useful to find an action principle for the threedimensional EW equations appeared for the first time in $[9,30]$. In particular, the suggestion to

\footnotetext{
${ }^{10}$ The action (54) is diffeomorphism-invariant by construction, since it can be written as $S=\int d^{3} x \sqrt{-g} \Psi$, where $\Psi$ transforms as a scalar under general coordinate transformations.
} 
use a CS term for the Weyl vector occurs as a final comment in [9]. (54) can also be written in the form

$$
\begin{aligned}
S & =\frac{1}{2 \kappa^{2}} \int d^{3} x\left[\sqrt{-g} f(R)+\frac{1}{2 \mu} \epsilon^{\mu \nu \rho} Q_{\rho} \hat{R}_{\nu \mu}\right]+\int d^{3} x \epsilon^{\mu \nu \rho}\left[\chi_{\rho \mu}\left(-\frac{1}{3} Q_{\nu}+\tilde{Q}_{\nu}\right)+\zeta_{\nu \sigma} T_{\rho \mu}{ }^{\sigma}\right] \\
& +\frac{3}{2 \kappa^{2} \mu} \int d^{3} x \epsilon^{\mu \nu \rho}\left(R_{\tau \mu \nu}^{\sigma} \Gamma_{\sigma \rho}^{\tau}-\frac{2}{3} \Gamma_{\sigma \mu}^{\tau} \Gamma_{\alpha \nu}^{\sigma} \Gamma_{\tau \rho}^{\alpha}\right) .
\end{aligned}
$$

From the variation of (54) w.r.t. $\chi_{\mu \nu}$ and $\zeta_{\mu \nu}$ we get respectively

$$
\begin{gathered}
\tilde{Q}_{\mu}=\frac{1}{3} Q_{\mu}, \\
\varepsilon^{\mu \rho \sigma} T_{\rho \sigma}{ }^{\nu}=0 .
\end{gathered}
$$

The latter implies vanishing torsion,

$$
T_{\rho \sigma}{ }^{\nu}=0 .
$$

Varying w.r.t $\Gamma^{\lambda} \mu \nu$ and using (50), (53), one obtains

$$
\begin{aligned}
& P_{\lambda}^{\mu \nu}+\delta_{\lambda}^{\nu} g^{\mu \sigma} \frac{\partial_{\sigma} f^{\prime}}{f^{\prime}}-g^{\mu \nu} \frac{\partial_{\lambda} f^{\prime}}{f^{\prime}}+\frac{2}{\mu f^{\prime}} \varepsilon^{\nu \rho \sigma}\left(3 R_{\lambda \rho \sigma}^{\mu}-\delta_{\lambda}^{\mu} \hat{R}_{\rho \sigma}\right) \\
& +\frac{2 \kappa^{2}}{f^{\prime}}\left(\delta_{\lambda}^{\nu} \varepsilon^{\mu \rho \sigma} \chi_{\rho \sigma}-\frac{2}{3} \delta_{\lambda}^{\mu} \varepsilon^{\nu \rho \sigma} \chi_{\rho \sigma}+g_{\lambda \tau} \varepsilon^{\tau \rho \sigma} \chi_{\rho \sigma} g^{\mu \nu}+\varepsilon^{\mu \nu \rho} \zeta_{\rho \lambda}\right)=0
\end{aligned}
$$

where $P_{\lambda}{ }^{\mu \nu}$ is the Palatini tensor defined in (16). In the following, we shall consistently set the traceless part of the nonmetricity to zero,

$$
\Omega_{\lambda \mu \nu}=0
$$

Notice that (56) and (60) are precisely the constraints needed to get the Weyl nonmetricity (1): Plugging (56) and (60) into the decomposition (47) gives $Q_{\lambda \mu \nu}=g_{\mu \nu} Q_{\lambda} / 3$, and thus

$$
\nabla_{\lambda} g_{\mu \nu}=-Q_{\lambda \mu \nu}=-\frac{1}{3} Q_{\lambda} g_{\mu \nu}
$$

which coincides with (1) for $h_{\lambda}=-Q_{\lambda} / 6$.

Using (56), (58), (60), and writing the Palatini tensor explicitly in terms of the Weyl vector $Q_{\mu},(59)$ becomes

$$
\begin{aligned}
& \frac{1}{6}\left(g^{\mu \nu} Q_{\lambda}-\delta_{\lambda}^{\nu} Q^{\mu}\right)+\delta_{\lambda}^{\nu} \frac{\partial^{\mu} f^{\prime}}{f^{\prime}}-g^{\mu \nu} \frac{\partial_{\lambda} f^{\prime}}{f^{\prime}}+\frac{6}{\mu f^{\prime}}\left[2 \varepsilon^{\alpha \nu \beta} g_{\lambda \alpha} \tilde{R}_{\beta}^{\mu}+\varepsilon^{\alpha \mu \nu}\left(g_{\alpha \lambda} \tilde{R}-2 \tilde{R}_{\alpha \lambda}\right)\right] \\
& +\frac{1}{\mu f^{\prime}}\left[\frac{1}{3} \varepsilon^{\alpha \mu \nu}\left(g_{\alpha \lambda} Q_{\rho} Q^{\rho}-Q_{\alpha} Q_{\lambda}+6 \tilde{\nabla}_{\alpha} Q_{\lambda}\right)+\varepsilon^{\alpha \nu \beta} g_{\alpha \lambda}\left(\frac{1}{3} Q_{\beta} Q^{\mu}-2 \tilde{\nabla}_{\beta} Q^{\mu}\right)\right] \\
& +\frac{2 \kappa^{2}}{f^{\prime}}\left(\delta_{\lambda}^{\nu} \varepsilon^{\mu \rho \sigma} \chi_{\rho \sigma}-\frac{2}{3} \delta_{\lambda}^{\mu} \varepsilon^{\nu \rho \sigma} \chi_{\rho \sigma}+g_{\lambda \tau} \varepsilon^{\tau \rho \sigma} \chi_{\rho \sigma} g^{\mu \nu}+\varepsilon^{\mu \nu \rho} \zeta_{\rho \lambda}\right)=0 .
\end{aligned}
$$

Here, $\tilde{\nabla}$ denotes the Levi-Civita connection, and $\tilde{R}_{\mu \nu}, \tilde{R}$ are respectively its Ricci tensor and scalar curvature. We also used (27), (28), (47) and (48) to express the Riemann tensor in terms of its Levi-Civita part and the Weyl vector. 
The $\lambda, \mu$ trace of (62) leads to

$$
\frac{2 \kappa^{2}}{f^{\prime}} \varepsilon^{\nu \rho \sigma} \zeta_{\rho \sigma}=0 \quad \Rightarrow \quad \zeta_{[\rho \sigma]}=0
$$

Taking this into account and considering the $\lambda, \nu$ trace of (62), we obtain

$$
\frac{10 \kappa^{2}}{3 f^{\prime}} \varepsilon^{\mu \rho \sigma} \chi_{\rho \sigma}-\frac{1}{6} Q^{\mu}-\frac{1}{\mu f^{\prime}} \varepsilon^{\mu \rho \sigma} \hat{R}_{\rho \sigma}+\frac{\partial^{\mu} f^{\prime}}{f^{\prime}}=0 .
$$

Plugging (63) and (64) into (62), and taking the $\mu, \nu$ trace of the resulting equation, one finds

$$
\chi_{\rho \sigma}=0,
$$

and thus (64) reduces to

$$
\frac{\partial^{\mu} f^{\prime}}{f^{\prime}}=\frac{1}{6} Q^{\mu}+\frac{1}{\mu f^{\prime}} \varepsilon^{\mu \rho \sigma} \hat{R}_{\rho \sigma} .
$$

With (65) and (66), (62) becomes

$$
\begin{aligned}
& \left(\delta_{\lambda}^{\nu} \varepsilon^{\mu \rho \sigma}-\varepsilon^{\alpha \rho \sigma} g_{\alpha \lambda} g^{\mu \nu}\right) \hat{R}_{\rho \sigma}+6\left[2 \varepsilon^{\alpha \nu \beta} g_{\lambda \alpha} \tilde{R}_{\beta}^{\mu}+\varepsilon^{\alpha \mu \nu}\left(g_{\alpha \lambda} \tilde{R}-2 \tilde{R}_{\lambda \alpha}\right)\right] \\
& +\frac{1}{3} \varepsilon^{\alpha \mu \nu}\left(g_{\alpha \lambda} Q_{\rho} Q^{\rho}-Q_{\alpha} Q_{\lambda}+6 \tilde{\nabla}_{\alpha} Q_{\lambda}\right)+\varepsilon^{\alpha \nu \beta} g_{\alpha \lambda}\left(\frac{1}{3} Q_{\beta} Q^{\mu}-2 \tilde{\nabla}_{\beta} Q^{\mu}\right)+2 \kappa^{2} \mu \varepsilon^{\mu \nu \rho} \zeta_{\lambda \rho}=0 .
\end{aligned}
$$

Before proceeding with the analysis of (67), consider the variation of (54) w.r.t. $g^{\mu \nu}$. To this end, observe that the Chern-Simons terms are topological, i.e., independent of the metric up to boundary terms. If we use moreover (60) and (65), it is straightforward to shew that the equations of motion of $g^{\mu \nu}$ are just given by the Einstein-Weyl equations (12) for $n=3$,

$$
R_{(\mu \nu)}-\frac{R}{3} g_{\mu \nu}=0 .
$$

Notice that, if one kept $\Omega_{\lambda \mu \nu} \neq 0$, there would be additional pieces in (68), so that one would not obtain precisely the EW equations, but something more general that contains a traceless part of the nonmetricity. Recall that (68) is supplemented by (56), (58) and (60), and that we get a nonexact Weyl vector whose dynamics is governed by (66). In terms of its Levi-Civita part and the Weyl vector, (68) reads

$$
\tilde{R}_{\mu \nu}-\frac{1}{3} g_{\mu \nu} \tilde{R}-\frac{1}{108} g_{\mu \nu} Q_{\rho} Q^{\rho}+\frac{1}{36} Q_{\mu} Q_{\nu}+\frac{1}{18} g_{\mu \nu} \tilde{\nabla}_{\rho} Q^{\rho}-\frac{1}{6} \tilde{\nabla}_{(\mu} Q_{\nu)}=0 .
$$

Plugging this into (67), the latter becomes

$$
\begin{aligned}
& \delta_{\lambda}{ }^{\nu} \varepsilon^{\mu \rho \sigma} \hat{R}_{\rho \sigma}-\varepsilon^{\alpha \rho \sigma} g_{\alpha \lambda} g^{\mu \nu} \hat{R}_{\rho \sigma}-2 \varepsilon^{\alpha \mu \nu} \hat{R}_{\lambda \alpha}+2 \varepsilon^{\alpha \nu \rho} g_{\alpha \lambda} \hat{R}_{\beta}^{\mu} \\
& +\varepsilon^{\alpha \mu \nu} g_{\alpha \lambda}\left(\frac{1}{9} Q_{\rho} Q^{\rho}-2 \tilde{R}+\frac{4}{3} \tilde{\nabla}_{\rho} Q^{\rho}\right)+2 \kappa^{2} \mu \varepsilon^{\mu \nu \rho} \zeta_{\lambda \rho}=0 .
\end{aligned}
$$

(66) is equivalent to

$$
\hat{R}^{\rho \sigma}=\varepsilon^{\rho \sigma \tau} \Upsilon_{\tau},
$$


where

$$
\Upsilon_{\tau}:=\frac{1}{2} \mu \varepsilon^{\rho \sigma \tau}\left(\frac{1}{6} f^{\prime} Q_{\tau}-\partial_{\tau} f^{\prime}\right) .
$$

Using this in (70), one easily shows that the terms involving the homothetic curvature tensor identically vanish, and we are left with

$$
\varepsilon^{\alpha \mu \nu} g_{\alpha \lambda}\left(\frac{1}{9} Q_{\rho} Q^{\rho}-2 \tilde{R}+\frac{4}{3} \tilde{\nabla}_{\rho} Q^{\rho}\right)+2 \kappa^{2} \mu \varepsilon^{\mu \nu \rho} \zeta_{\lambda \rho}=0
$$

or equivalently

$$
\zeta_{\mu \nu}=\frac{1}{\kappa^{2} \mu} g_{\mu \nu}\left(\tilde{R}-\frac{1}{18} Q_{\rho} Q^{\rho}-\frac{2}{3} \tilde{\nabla}_{\rho} Q^{\rho}\right)=\frac{1}{\kappa^{2} \mu} g_{\mu \nu} R,
$$

where we used (56), (58) and (60) in the last step. Summarizing, one has

$$
Q_{\lambda \mu \nu}=\frac{1}{3} Q_{\lambda} g_{\mu \nu}
$$

which corresponds to Weyl nonmetricity, together with (58), (66) and (68). The latter are precisely the Einstein-Weyl equations in three dimensions. The final form of the connection, obtained by plugging (58) and (75) into (28), results to be

$$
\Gamma_{\mu \nu}^{\lambda}=\tilde{\Gamma}_{\mu \nu}^{\lambda}-\frac{1}{6} g_{\mu \nu} Q^{\lambda}+\frac{1}{3} \delta_{(\mu}^{\lambda} Q_{\nu)} .
$$

With $f(R)=R^{3 / 2}$, eq. (66) becomes

$$
\partial_{\mu} \ln R=\frac{1}{3} Q_{\mu}+\frac{4}{3 \mu \sqrt{R}} g_{\mu \tau} \varepsilon^{\rho \sigma \tau} \hat{R}_{\rho \sigma} .
$$

As we can see, the Weyl vector is not exact and possesses a nontrivial dynamics. In particular, (77) is a differential equation containing only the Weyl vector and the metric. Dualizing (77) gives the generalized monopole equation (cf. [13]),

$$
d h=\star(d \Sigma+h \Sigma),
$$

with the one-form $h$ and the function $\Sigma$ respectively defined by $h_{\lambda}=-Q_{\lambda} / 6$ and $\Sigma=\mu \sqrt{R} / 4$. Actually, (78) represents a special case of the generalized monopole equation, since the latter has the exterior derivative of any one-form $\omega$ on the lhs. If $\Sigma$ were constant (this can always be achieved by a Weyl rescaling (10), under which $\Sigma \mapsto e^{-\Omega} \Sigma$ ), (78) would boil down to $d h=\star h \Sigma$, which is the self-duality condition (3) of [31] in three dimensions. We can thus regard (78) as a conformally invariant generalization of the three-dimensional self-duality condition.

\section{Final remarks}

A longstanding open mathematical problem has been the construction of an action principle for the Einstein-Weyl equations. In this paper, we presented for the first time such an action in three dimensions, given by a metric affine $f(R)$ gravity contribution plus additional pieces involving Lagrange multipliers and gravitational Chern-Simons terms. To be more precise, our 
model contains, in addition to the Weyl nonmetricity, also a traceless part. However, the latter can be consistently set to zero, and in this case our equations of motion boil down to the EW equations.

Let us spend some words on the matter coupling of (54). As mentioned in section 2, when one adds matter to metric affine $f(R)$ theories of gravity, the projective invariance of the action imposes the constraint $\Delta_{\mu}{ }^{\mu \nu}=0$ on the hypermomentum. However, if we couple matter to the theory developed in the present paper, we see that the extra contributions we have introduced in the action break projective invariance. Thus, in particular, $\Delta_{\mu}{ }^{\mu \nu} \neq 0$, and the inconsistency mentioned above does not appear anymore.

It remains to be seen if our results can be extended to higher dimensions. If so, this would probably involve topological terms like e.g. $B F$ actions, in addition to $f(R)$ gravity. Note that Chern-Simons terms were considered in modifications of four-dimensional general relativity [32-34]. We hope to come back to this point in a future publication.

Finally, it would also be interesting to explore possible cosmological applications of (54), along the lines of refs. [35,36], which are based on $f(R)$ gravity theories with a Levi-Civita connection. In this context, [37] presented a model for cosmological hyperfluids, i.e., fluids with intrinsic hypermomentum that induce spacetime torsion and nonmetricity.

\section{Acknowledgements}

This work was supported partly by INFN and by MIUR-PRIN contract 2017CC72MK003. The authors would like to thank L. Andrianopoli, R. D'Auria and M. Trigiante for inspiring discussions.

\section{References}

[1] R. Adler, M. Bazin, M. Schiffer, "Introduction to general relativity," Mc.Graw-Hill 1965.

[2] D. M. J. Calderbank and H. Pedersen, "Einstein-Weyl geometry," in Essays on Einstein Manifolds (eds. C. R. LeBrun and M. Wang), Surveys in Differential Geometry, vol. V, International Press.

[3] G. B. Folland, "Weyl manifolds," J. Diff. Geom. 4 (1970) 145.

[4] C. Romero, J. B. Fonseca-Neto and M. L. Pucheu, "General relativity and Weyl geometry," Class. Quant. Grav. 29 (2012) 155015 [arXiv:1201.1469 [gr-qc]].

[5] H. Weyl, "Gravitation and electricity," Sitzungsber. Preuss. Akad. Wiss. Berlin (Math. Phys.) 1918 (1918) 465.

[6] H. Weyl, "A new extension of relativity theory," Annalen Phys. 59 (1919) 101 [Surveys High Energ. Phys. 5 (1986) 237] [Annalen Phys. 364 (1919) 101].

[7] J. T. Wheeler, "Weyl geometry," Gen. Rel. Grav. 50 (2018) no.7, 80 [arXiv:1801.03178 [gr-qc]].

[8] N. Hitchin, "Complex manifolds and Einstein's equations," Lect. Notes Math. 970 (1982), 73.

[9] H. Pedersen and K. P. Tod, "Three-dimensional Einstein-Weyl geometry," Adv. Math. 97 (1993), 74 . 
[10] R. Ward, "Einstein-Weyl spaces and SU( $\infty$ ) Toda fields," Class. Quant. Grav. 7 (1990), L95L98.

[11] M. Dunajski, L. Mason and P. Tod, "Einstein-Weyl geometry, the dKP equation and twistor theory," J. Geom. Phys. 37 (2001), 63 [arXiv:math/0004031 [math.DG]].

[12] M. Dunajski, "An interpolating dispersionless integrable system," J. Phys. A 4 (2008), no. 31, 315202.

[13] P. Jones and K. Tod, "Minitwistor spaces and Einstein-Weyl spaces," Class. Quant. Grav. 2 (1985) no.4, 565.

[14] P. Gauduchon and K. P. Tod, "Hyper-Hermitian metrics with symmetry," J. Geom. Phys. 25 (1998), 291.

[15] P. Meessen and A. Palomo-Lozano, "Cosmological solutions from fake $N=2$ EYM supergravity," JHEP 05 (2009), 042 [arXiv:0902.4814 [hep-th]].

[16] J. Gutowski and W. Sabra, "Solutions of minimal four-dimensional de Sitter supergravity," Class. Quant. Grav. 27 (2010), 235017 [arXiv:0903.0179 [hep-th]].

[17] J. Grover, J. B. Gutowski, C. A. Herdeiro, P. Meessen, A. Palomo-Lozano and W. A. Sabra, "Gauduchon-Tod structures, Sim holonomy and de Sitter supergravity," JHEP 07 (2009), 069 [arXiv:0905.3047 [hep-th]].

[18] M. Dunajski, J. Gutowski, W. Sabra and P. Tod, "Cosmological Einstein-Maxwell instantons and Euclidean supersymmetry: Anti-self-dual solutions," Class. Quant. Grav. 28 (2011), 025007 [arXiv:1006.5149 [hep-th]].

[19] M. Dunajski, J. Gutowski, W. Sabra and P. Tod, "Cosmological Einstein-Maxwell instantons and Euclidean supersymmetry: Beyond self-duality," JHEP 03 (2011), 131 [arXiv:1012.1326 [hep-th]].

[20] M. Dunajski, J. Gutowski and W. Sabra, "EinsteinWeyl spaces and near-horizon geometry," Class. Quant. Grav. 34 (2017) no.4, 045009 [arXiv:1610.08953 [hep-th]].

[21] S. Klemm and L. Ravera, "Supersymmetric near-horizon geometry and Einstein-Cartan-Weyl spaces," Phys. Lett. B 793 (2019), 265 [arXiv:1904.03681 [hep-th]].

[22] L. Ciambelli and R. G. Leigh, "Weyl connections and their role in holography," Phys. Rev. D 101 (2020) no.8, 086020 [arXiv:1905.04339 [hep-th]].

[23] S. Klemm and L. Ravera, "Einstein manifolds with torsion and nonmetricity," Phys. Rev. D 101 (2020) no.4, 044011 [arXiv:1811.11458 [gr-qc]].

[24] F. W. Hehl, J. D. McCrea, E. W. Mielke and Y. Ne'eman, "Metric-affine gauge theory of gravity: Field equations, Noether identities, world spinors, and breaking of dilation invariance," Phys. Rept. 258 (1995) 1 [gr-qc/9402012].

[25] D. Iosifidis, A. C. Petkou and C. G. Tsagas, "Torsion/nonmetricity duality in $f(R)$ gravity," Gen. Rel. Grav. 51 (2019) no.5, 66 [arXiv:1810.06602 [gr-qc]]. 
[26] D. Iosifidis, "Metric-affine gravity and cosmology/Aspects of torsion and nonmetricity in gravity theories," arXiv:1902.09643 [gr-qc].

[27] T. P. Sotiriou and S. Liberati, "Metric-affine $f(R)$ theories of gravity," Annals Phys. 322 (2007) 935 [gr-qc/0604006].

[28] T. P. Sotiriou and S. Liberati, "The metric-affine formalism of $f(R)$ gravity," J. Phys. Conf. Ser. 68 (2007) 012022 [gr-qc/0611040].

[29] V. Vitagliano, T. P. Sotiriou and S. Liberati, "The dynamics of metric-affine gravity," Annals Phys. 326 (2011) 1259 Erratum: [Annals Phys. 329 (2013) 186] [arXiv:1008.0171 [gr-qc]].

[30] S. L. Cacciatori, M. M. Caldarelli, A. Giacomini, S. Klemm and D. S. Mansi, "Chern-Simons formulation of three-dimensional gravity with torsion and nonmetricity," J. Geom. Phys. 56 (2006), 2523 [arXiv:hep-th/0507200 [hep-th]].

[31] P. Townsend, K. Pilch and P. van Nieuwenhuizen, "Selfduality in odd dimensions," Phys. Lett. B 136 (1984), 38.

[32] R. Jackiw and S. Y. Pi, "Chern-Simons modification of general relativity," Phys. Rev. D 68 (2003) 104012 [gr-qc/0308071].

[33] S. Alexander and N. Yunes, "Chern-Simons modified general relativity," Phys. Rept. 480 (2009) 1 [arXiv:0907.2562 [hep-th]].

[34] F. W. Hehl, J. D. McCrea, W. Kopczynski and E. W. Mielke, "Chern-Simons terms in metricaffine space-time: Bianchi identities as Euler-Lagrange equations," J. Math. Phys. 32 (1991) 2169.

[35] S. Capozziello, C. A. Mantica and L. G. Molinari, "Cosmological perfect fluids in Gauss-Bonnet gravity," Int. J. Geom. Meth. Mod. Phys. 16 (2019) no. 09, 1950133 [arXiv:1906.05693 [gr-qc]].

[36] S. Capozziello, C. A. Mantica and L. G. Molinari, "Cosmological perfect fluids in higher-order gravity," Gen. Rel. Grav. 52 (2020) no.4, 36 [arXiv:1908.10176 [gr-qc]].

[37] D. Iosifidis, "Cosmological hyperfluids, torsion and nonmetricity," [arXiv:2003.07384 [gr-qc]]. 